\title{
GROUPS OF EMBEDDED MANIFOLDS
}

\author{
BY \\ MAX K. AGOSTON
}

\begin{abstract}
This paper defines a group $\theta\left(M^{n}, v_{\varphi}\right)$ which generalizes the group of framed homotopy $n$-spheres in $S^{n+k}$. Let $M^{n}$ be an arbitrary 1-connected manifold satisfying a weak condition on its homology in the middle dimension and let $\nu_{\varphi}$ be the normal bundle of some imbedding $\varphi: M^{n} \rightarrow S^{n+k}$, where $2 k \geqq n+3$. Then $\theta\left(M^{n}, \nu_{\varphi}\right)$ is the set of $h$-cobordism classes of triples $\left(F, V^{n}, f\right)$, where $F: S^{n+k} \rightarrow T\left(v_{\oplus}\right)$ is a map which is transverse regular on $M, V^{n}=F^{-1}\left(M^{n}\right)$, and $f=F \mid V^{n}$ is a homotopy equivalence. $\left(T\left(v_{\varphi}\right)\right.$ is the Thom complex of $\nu_{\varphi}$.) There is a natural group structure on $\theta\left(M^{n}, v_{\varphi}\right)$, and $\theta\left(M^{n}, v_{\varphi}\right)$ fits into an exact sequence similar to that for the framed homotopy $n$-spheres.
\end{abstract}

This paper attempts to generalize in a natural way a well-known exact sequence concerning framed homotopy spheres which is contained in the work of Novikov [11], Kervaire-Milnor [7], and Levine [10]. The author stumbled onto these results partly because of his efforts to prove imbedding theorems for manifolds in the metastable range, and partly because of his recent work on Browder-Novikov theory for maps of degree $d,|d| \neq 0$ (see [2]).

$\$ 2$ describes the basic constructions used in this paper. The "group of embedded manifolds", $\theta\left(M, v_{\varphi}\right)$, is defined in $\S 3$. A fairly simple description of that group is given towards the end of that section. $\$ 3$ also contains the main results about $\theta\left(M, v_{\varphi}\right)$. In $\S 4$ we discuss a few interesting open problems. The author would like to thank the referee for some helpful suggestions.

1. Notation. All manifolds will be $C^{\infty}$, compact, and oriented. Maps will be transverse to boundaries.

If $M^{n}$ is a connected closed manifold, let $[M] \in H_{n} M$ denote the orientation class. Recall that $f: V^{n} \rightarrow M^{n}$ is said to have degree $d$, i.e., $\operatorname{deg} f=d$, if $f_{*}([V])$ $=d[M]$, where $f_{*}: H_{n} V \rightarrow H_{n} M$ is the map induced by $f$ on the integral homology groups.

As usual, $D^{k}$ denotes the closed unit ball in Euclidean $k$-space $\boldsymbol{R}^{k}$, i.e., $D^{k}=\left\{\left(y_{1}, \ldots, y_{k}\right) \in \boldsymbol{R}^{k} \mid y_{1}^{2}+\cdots+y_{k}^{2} \leqq 1\right\} . \quad S^{k}=\partial D^{k+1}=D_{+}^{k} \cup D_{-}^{k}, \quad$ where $D_{+}^{k}$ $=\left\{\left(y_{1}, \ldots, y_{k+1}\right) \in \boldsymbol{R}^{k+1} \mid y_{1}^{2}+\cdots+y_{k+1}^{2}=1, y_{1} \geqq 0\right\} \quad$ and $\quad D_{-}^{k}=\left\{\left(y_{1}, \ldots, y_{k+1}\right)\right.$ $\left.\in \boldsymbol{R}^{k+1} \mid y_{1}^{2}+\cdots+y_{k+1}^{2}=1, y_{1} \leqq 0\right\}$. We have natural inclusions $S^{k} \subseteq S^{k+1}$ and $D^{k} \subseteq D^{k+1}$. Let $e=(1,0) \in S^{0} \subseteq S^{k}$.

Received by the editors September 5, 1969.

AMS 1970 subject classifications. Primary 57D55, 57D99; Secondary 57D60.

Key words and phrases. Framed homotopy $n$-spheres in $S^{n+k}$, normally equivalent $n$ manifolds in $S^{n+k}$, exact sequence. 
If $f: V^{n} \rightarrow W^{n+q}$ is an imbedding, we shall consider $f$ as an inclusion map and identify the total space $E=E\left(\nu_{f}\right)$ of the normal disk bundle $\nu_{f}$ with a tubular neighborhood of $V$ in $W . T\left(\nu_{f}\right)=E / \partial E$ is the Thom complex of $\nu_{f}$, and $T_{f}: W \rightarrow T\left(\nu_{f}\right)$ is the natural collapsing map. Given $g: U^{m} \rightarrow T\left(\nu_{f}\right)$ which is transverse regular on $V$ so that $N=g^{-1}(V)$ is an $(m-q)$-submanifold of $U$, we shall always assume that a tubular neighborhood $T$ of $N$ has been given a fixed bundle structure which is the pullback of $\nu_{f}$ under $g$. We also assume that $N$ is given an orientation which is induced from the orientation of $V$.

If $V^{n} \subseteq W^{n+q}$, then by a framing of $V$ in $W$, or by a framing of a tubular neighborhood $T$ of $V$, we shall mean a diffeomorphism $\mathscr{F}: V \times D^{q} \rightarrow T$ such that $\mathscr{F}(x, 0)=x$. Two framed submanifolds $\left(V_{1}^{n}, \mathscr{F}_{1}\right)$ and $\left(V_{2}^{n}, \mathscr{F}_{2}\right)$ in $W^{n+q}$ are framed cobordant if there is a framed submanifold $\left(N^{n+1}, \mathscr{G}\right)$ in $W \times[1,2]$ such that $(N, \mathscr{G}) \cap(W \times i)=\left(V_{i}, \mathscr{F}_{i}\right) \times i, i=1,2$. They are framed $h$-cobordant if $N$ is an $h$-cobordism.

2. Preliminaries. Throughout this paper we shall make the following assumptions: $M^{n}$ is a 1 -connected oriented manifold with $n \geqq 5$. Let $t=[n / 2]$. Then either $n \equiv 0(\bmod 4)$, or $H_{t} M=0$ and $H_{t-1} M$ is torsion-free. $\varphi$ is an imbedding of $M$ in $S^{n+k}$, where $2 k \geqq n+3$.

DEFINITION.

$\mathscr{T}_{0}^{+}\left(M, \nu_{\varphi}\right)=\left\{\left(F, V^{n}, f\right) \mid F: S^{n+k} \rightarrow T\left(v_{\varphi}\right)\right.$ is a map which is transverse regular on $M$ with $V^{n}=F^{-1}(M) 1$-connected and $\left.f=F \mid V: V \rightarrow M\right\}$.

$\mathscr{S}_{0}{ }^{+}\left(M, v_{\varphi}\right)=\left\{(F, V, f) \in \mathscr{T}_{0}^{+}\left(M, v_{\varphi}\right) \mid \operatorname{deg} f>0\right.$ and $f_{*}: H_{i} V \rightarrow H_{i} M$

is an isomorphism for $0 \leqq i \leqq[n / 2]\}$.

NoTE. $\mathscr{S}_{0}^{+}\left(M, \nu_{\varphi}\right) \neq \varnothing$ since it contains $\left(T_{\varphi}, M\right.$, identity).

If $\alpha_{i}=\left(F_{i}, V_{i}, f_{i}\right) \in \mathscr{T}_{0}^{+}\left(M, \nu_{\varphi}\right)$, define $\alpha_{1} \sim \alpha_{2}$ if there is a map $H: S^{n+k} \times[1,2]$ $\rightarrow T\left(v_{\varphi}\right)$ which is transverse regular on $M$ such that $H \mid S^{n+k} \times i=F_{i} \times i$ and $H^{-1}(M)$ is an $h$-cobordism between $V_{1} \times 1$ and $V_{2} \times 2$. Clearly, $\sim$ is an equivalence relation. We set

$$
\mathscr{T}^{+}\left(M, v_{\varphi}\right)=\mathscr{T}_{0}^{+}\left(M, v_{\varphi}\right) / \sim \text { and } \mathscr{S}^{+}\left(M, v_{\varphi}\right)=\mathscr{S}_{0}^{+}\left(M, v_{\varphi}\right) / \sim \subseteq \mathscr{T}^{+}\left(M, v_{\varphi}\right) \text {. }
$$

If $\alpha \in \mathscr{T}_{0}^{+}\left(M, v_{\varphi}\right)$, we shall also write $\alpha$ for the equivalence class that $\alpha$ determines in $\mathscr{T}^{+}\left(M, \nu_{\varphi}\right)$.

Suppose that $\alpha_{i}=\left(F_{i}, V_{i}, f_{i}\right) \in \mathscr{T}^{+}\left(M, \nu_{\varphi}\right)$. Let $\nu_{i}$ be the normal disk bundle of $V_{i}$ in $S^{n+k}$ and let $D_{+}^{n} \times D^{k}$ and $D_{-}^{n} \times D^{k}$ be canonical tubular neighborhoods of $D_{+}^{n}$ and $D_{-}^{n}$ in $D_{+}^{n+k}$ and $D_{-}^{n+k}$, respectively. Without loss of generality we may assume that $D_{+}^{n} \subseteq V_{1}, \quad D_{-}^{n} \subseteq V_{2}, E\left(\nu_{1} \mid S^{n-1}\right)=E\left(\nu_{2} \mid S^{n-1}\right) \subseteq S^{n+k-1}, \quad V_{1}-D_{+}^{n} \subseteq D_{-}^{n} \times D^{k}$, $V_{2}-D_{-}^{n} \subseteq D_{+}^{n} \times D^{k}, f_{1}\left(D_{+}^{n}\right)=f_{2}\left(D_{-}^{n}\right)=x_{0} \in M$, and $f_{1}\left|S^{n+k-1}=f_{2}\right| S^{n+k-1}$. Now define $F_{3}: S^{n+k} \rightarrow T\left(\nu_{\varphi}\right)$ by $F_{3}\left|D_{+}^{n+k}=f_{2}\right| D_{+}^{n+k}$ and $F_{3}\left|D_{-}^{n+k}=f_{1}\right| D_{-}^{n+k}$. Let $V_{3}$ $=F_{3}^{-1}(M), f_{3}=F_{3} \mid V_{3}$, and $\alpha_{1} \# \alpha_{2}=\left(F_{3}, V_{3}, f_{3}\right)$. Then $\operatorname{deg} f_{3}=\operatorname{deg} f_{1}+\operatorname{deg} f_{2}, V_{3}$ $=V_{1} \# V_{2}$, and $\alpha_{1} \# \alpha_{2}$ is a well defined element of $\mathscr{T}^{+}\left(M, \nu_{\varphi}\right)$. 
Next, define $\theta_{f}^{n+k, n}$ to be the group of $h$-cobordism classes of framed homotopy $n$-spheres in $S^{n+k}$. If $\Sigma^{n}$ is a homotopy $n$-sphere in $S^{n+k}$ with a framing $\mathscr{F}$ of its normal disk bundle $\nu_{\Sigma}$, we let $[\Sigma, \mathscr{F}]$ denote the element it determines in $\theta_{f}^{n+k, n}$. (Note that in analogy with $\theta_{f}^{n+k, n}$ we can think of $\mathscr{T}^{+}\left(M, \nu_{\varphi}\right)$ and $\mathscr{S}^{+}\left(M, \nu_{\varphi}\right)$ as $h$-cobordism classes of certain submanifolds $V^{n} \subseteq S^{n+k}$ with a given bundle map from the normal disk bundle of $V$ in $S^{n+k}$ to $\nu_{\varphi}$.)

Keeping the notation of the two previous paragraphs, let $\sigma=[\Sigma, \mathscr{F}] \in \theta_{f}^{n+k, n}$. Assume that $D_{-}^{n} \subseteq \Sigma, E\left(\nu_{\Sigma} \mid S^{n-1}\right)=E\left(\nu_{1} \mid S^{n-1}\right)$, and $X=\Sigma-D_{-}^{n} \subseteq D_{+}^{n} \times D^{k}$. Define $F^{\prime}: S^{n+k} \rightarrow T\left(\nu_{\varphi}\right)$ by $F^{\prime}\left|D_{-}^{n+k}=f_{1}\right| D_{-}^{n+k}, F^{\prime}(\mathscr{F}(y, u))=f_{1}(\mathscr{F}(e, u))$, for $(y, u) \in X \times D^{k}$, and $F^{\prime}\left(D_{-}^{n+k}-\mathscr{F}\left(X \times D^{k}\right)\right)=$ canonical base point of $T\left(\nu_{\varphi}\right)$. Set $V^{\prime}=\left(F^{\prime}\right)^{-1}(M)$, $f^{\prime}=F^{\prime} \mid V^{\prime}$, and $\alpha_{1} \# \sigma=\left(F^{\prime}, V^{\prime}, f^{\prime}\right)$. Then $\alpha_{1} \# \sigma$ is a well defined element of $\mathscr{T}^{+}\left(M, \nu_{\varphi}\right), \operatorname{deg} f^{\prime}=\operatorname{deg} f$, and $V^{\prime}=V_{1} \# \Sigma$.

One can easily check that if $\alpha_{i} \in \mathscr{T}^{+}\left(M, \nu_{\varphi}\right)$ and $\sigma_{i} \in \theta_{f}^{n+k, n}$, then the two connected sum operations defined above have the following properties:

(1) $\left(\alpha_{1} \# \alpha_{2}\right) \# \alpha_{3}=\alpha_{1} \#\left(\alpha_{2} \# \alpha_{3}\right)$,

(2) $\alpha_{1} \# \alpha_{2}=\alpha_{2} \# \alpha_{1}$,

(3) $\left(\alpha_{1} \# \alpha_{2}\right) \# \sigma_{1}=\alpha_{1} \#\left(\alpha_{2} \# \sigma_{1}\right)$,

(4) $\left(\alpha_{1} \# \sigma_{1}\right) \# \alpha_{2}=\left(\alpha_{1} \# \alpha_{2}\right) \# \sigma_{1}$,

(5) $\alpha_{1} \#\left(\sigma_{1}+\sigma_{2}\right)=\left(\alpha_{1} \# \sigma_{1}\right) \# \sigma_{2}$, and

(6) if $\alpha_{1} \in \mathscr{S}^{+}\left(M, v_{\varphi}\right)$, then $\alpha_{1} \# \sigma_{1} \in \mathscr{S}^{+}\left(M, v_{\varphi}\right)$.

Lemma 1. Let $\alpha_{i}=\left(F_{i}, V_{i}, f_{i}\right) \in \mathscr{S}^{+}\left(M, \nu_{\varphi}\right)$ and suppose $\alpha_{1} \# \alpha_{2}=\left(F_{3}, F_{3}, f_{3}\right)$. Then there exists a triple $\Gamma=\left(H, W^{n+1}, h\right)$ such that

(a) $H: S^{n+k} \times[3,4] \rightarrow T\left(\nu_{\varphi}\right)$ is a map which is transverse regular on $M$ with $W=H^{-1}(M)$ 1-connected and $h=H \mid W: W \rightarrow M$;

(b) $H \mid S^{n+k} \times l=F_{l} \times l, l=3,4$;

(c) $\alpha_{4}=\left(F_{4}, V_{4}, f_{4}\right) \in \mathscr{S}^{+}\left(M, v_{\varphi}\right)$ where $V_{4}=F_{4}^{-1}(M)$ and $f_{4}=F_{4} \mid V_{4}$; and

(d) $H_{i}\left(W, V_{4}\right)=0$ for $i \geqq t+1$ if $n \neq 0(\bmod 4)$.

Proof. We shall define inductively a sequence $\Gamma_{i}=\left(H_{i}, W_{i}, h_{i}\right)$, for $0 \leqq i \leqq[n / 2]-1$, such that

(1) $H_{i}: S^{n+k} \times[0, i+1] \rightarrow T\left(v_{\varphi}\right)$ is a map which is transverse regular on $M$ with $W_{i}=H_{i}^{-1}(M) 1$-connected and $h_{i}=H_{i} \mid W_{i}$;

(2) $H_{i} \mid S^{n+k} \times[0, i]=H_{i-1}$;

(3) $\partial W_{i}=V_{3} \times 0 \cup N_{i}$ with $N_{i}$ 1-connected;

(4) $\left(h_{i}\right)_{*}: H_{t} W_{i} \rightarrow H_{t} M$ is an isomorphism for $0 \leqq t \leqq i$;

(5) if $j: V_{3} \times 0 \rightarrow W_{i}$ is the natural inclusion, then $j_{*}: H_{t}\left(V_{3} \times 0\right) \rightarrow H_{t} W_{i}$ is an isomorphism for $t>i+1$; if $t=i+1, j_{*}$ is one-to-one and $H_{i+1} W_{i}=j_{*} H_{i+1}\left(V_{3} \times 0\right)$ $\oplus G$, where $G$ is a torsion-free group which is zero if $H_{i} M$ had no torsion and $\left(h_{i}\right)_{*}(G)=0$.

Define $H_{0}: S^{n+k} \times[0,1] \rightarrow T\left(v_{\varphi}\right)$ by $H_{0}(x, t)=F_{3}(x)$. Then $H_{0}$ clearly determines a triple $\Gamma_{0}=\left(H_{0}, W_{0}, h_{0}\right)$ which satisfies (1)-(5). Suppose $\Gamma_{i-1}=\left(H_{i-1}, W_{i-1}, h_{i-1}\right)$ 
has been defined for $1 \leqq i \leqq[n / 2]-1$ satisfying (1)-(5). Our object will be to add handles to $W_{i-1}$ along $N_{i-1}$ to make $h_{i-1} i$-connected.

Let $j^{\prime}: N_{i-1} \rightarrow W_{i-1}$ be the natural inclusion and consider the exact sequence

$$
\begin{aligned}
& \cdots \longrightarrow H_{i+1}\left(W_{i-1}, N_{i-1}\right) \longrightarrow H_{i} N_{i-1} \underset{j_{*}^{\prime}}{\longrightarrow} H_{i} W_{i-1}=j_{*} H_{i}\left(V_{3} \times 0\right) \oplus G \rightarrow \cdots \\
& H^{n-i}\left(W_{i-1}, V_{3} \times 0\right)
\end{aligned}
$$

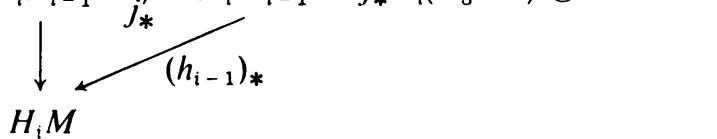

Since $i \leqq[n / 2]-1$, the universal coefficient theorem for cohomology and (5) imply that $j_{*}^{\prime}$ is an isomorphism. Let $K_{i}=$ kernel of $\left(f_{3}\right)_{*}: H_{i} V_{3} \rightarrow H_{i} M . K_{i}$ is a direct summand of $H_{i} V_{3}$ because $V_{3}=V_{1} \# V_{2}$ and $\left(f_{l}\right)_{*}: H_{i} V_{l} \rightarrow H_{i} M, l=1,2$, is an isomorphism. Therefore, if $K=$ kernel of $\left(h_{i-1}\right)_{*}: H_{i} W_{i-1} \rightarrow H_{i} M$, then $K$ is a direct summand of $H_{i} W_{i-1}$ and $K=j_{*}\left(K_{i}\right) \oplus G$. The diagram

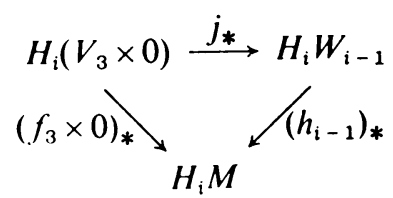

shows that $\left(h_{i-1}\right)_{*}$ is onto $H_{i} M$. It follows that every element of $K$ can be realized as an imbedded sphere in $N_{i-1}$ with trivial normal bundle. One can now add handles to $W_{i-1}$ along $N_{i-1}$ to kill $K$ as in the case of the usual Browder-Novikov theory. In fact, since $2 k \geqq n+1$, the handles can be attached in $S^{n+k} \times i$ so that the method of [4] and [9] can be used to obtain $\Gamma_{i}=\left(H_{i}, W_{i}, h_{i}\right) . \Gamma_{i}$ will satisfy (1)-(4) trivially. The proof of Theorem 2.1 in [1] shows how (5) can be satisfied also. This finishes the inductive definition of $\Gamma_{i}$.

Let $t=[n / 2]$. Assume that $n \not \equiv 0(\bmod 4)$. By hypothesis $H_{t} M=0$ and $H_{t-1} M$ is torsion-free, and so (5) implies that $\left(h_{t-1} \mid N_{t-1}\right)_{*}: H_{l} N_{t-1} \rightarrow H_{l} M$ is an isomorphism for $0 \leqq l \leqq[n / 2]$. Define $H: S^{n+k} \times[3,4] \rightarrow T\left(\nu_{\varphi}\right)$ by $H(x, u)=H_{t-1}(x, t(u-3))$ and set $W=H^{-1}(M), h=H \mid W$. Then $\Gamma=(H, W, h)$ satisfies (a)-(d) in Lemma 1 and the lemma is proved in this case. If $n \equiv 0(\bmod 4)$, then there is no obstruction to doing surgery on $N_{t-1}$ in the middle dimension and one can define $\Gamma_{t}$ satisfying (1)-(4) very much like the other $\Gamma_{i}$. Therefore we can get a $\Gamma$ satisfying (a)-(c) in this case also. This completes the proof of Lemma 1.

We can now define an operation + in $\mathscr{S}^{+}\left(M, \nu_{\varphi}\right)$ as follows: If $\alpha_{i} \in \mathscr{S}^{+}\left(M, \nu_{\varphi}\right)$, then we let $\alpha_{1}+\alpha_{2}=\alpha_{4}$, where $\alpha_{4}$ is defined as in Lemma 1(c).

LEMMA 2. + is a well defined associative and commutative operation.

Proof. Suppose that $\Gamma^{\prime}=\left(H^{\prime}, W^{\prime}, h^{\prime}\right)$ and $\alpha_{4}^{\prime}=\left(F_{4}^{\prime}, V_{4}^{\prime}, f_{4}^{\prime}\right)$ are triples which satisfy (a)-(d) in Lemma 1. + will be well defined once we show that $\alpha_{4}^{\prime}=\alpha_{4}$. Define $H^{\prime \prime}: S^{n+k} \times[2,4] \rightarrow T\left(\nu_{\varphi}\right)$ by $H^{\prime \prime}(x, u)=H^{\prime}(x,-u+6)$ for $u \in[2,3]$ and $H^{\prime \prime}(x, u)=H(x, u)$ for $u \in[3,4]$. Let $W^{\prime \prime}=\left(H^{\prime \prime}\right)^{-1}(M)$ and $h^{\prime \prime}=H^{\prime \prime} \mid W^{\prime \prime}$. It suffices to show that we can make $W^{\prime \prime}$ into an $h$-cobordism via framed surgery in $S^{n+k} \times$ 
$(2,4)$. To be precise, we are looking for a map $P: S^{n+k} \times[2,4] \times[0,1] \rightarrow T\left(\nu_{\varphi}\right)$ satisfying

(1) $P$ is transverse regular on $M$;

(2) $P\left|S^{n+k} \times[2,4] \times 0=H^{\prime \prime} \times 0, \quad P\right| S^{n+k} \times 2 \times u=F_{4}^{\prime} \times 2 \times u, \quad P \mid S^{n+k} \times 4 \times u=$ $F_{4} \times 4 \times u$, for $u \in[0,1]$; and

(3) $U=P^{-1}(M) \cap S^{n+k} \times[2,4] \times 1$ is an $h$-cobordism.

If $n \equiv 0(\bmod 4)$, then there is no obstruction to doing surgery on $W^{\prime \prime}$, even in the middle dimensions. If $n \neq 0(\bmod 4)$, then it follows from Lemma 1(d) (using our hypothesis on the homology of $M$ in the middle dimensions) that we only have to do surgery on $W^{\prime \prime}$ in dimension $\leqq[n / 2]$. In any case, it is therefore possible to define $P$ inductively, similar to the definition of the $\Gamma_{i}$ in the proof of Lemma 1. We shall omit the details and leave it to the reader to translate the construction for the $\Gamma_{i}$ so that it is applicable in this situation.

Finally, the fact that + is associative and commutative follows from the fact that \# has these properties. This finishes the proof of Lemma 2.

Lemma 3. Let $\alpha_{i} \in \mathscr{S}^{+}\left(M, v_{\varphi}\right)$ and $\sigma_{i} \in \theta_{f}^{n+k, n}$. Then

$$
\left(\alpha_{1} \#\left(\sigma_{1}+\sigma_{2}\right)\right)+\alpha_{2}=\left(\alpha_{1} \# \sigma_{1}\right)+\left(\alpha_{2} \# \sigma_{2}\right) .
$$

Proof. This lemma is an easy consequence of the observation that

$$
\left(\alpha_{1} \#\left(\sigma_{1}+\sigma_{2}\right)\right) \# \alpha_{2}=\left(\alpha_{1} \# \sigma_{1}\right) \#\left(\alpha_{2} \# \sigma_{2}\right) .
$$

Next, let $\alpha=(F, V, f) \in \mathscr{T}^{+}\left(M, v_{\varphi}\right)$. Define

$$
\psi_{0}^{\prime}: \mathscr{T}^{+}\left(M, \nu_{\varphi}\right) \rightarrow \pi_{n+k} T\left(\nu_{\varphi}\right) \quad \text { and } \quad \psi_{0}: \mathscr{S}^{+}\left(M, \nu_{\varphi}\right) \rightarrow \pi_{n+k} T\left(v_{\varphi}\right)
$$

by $\psi_{0}^{\prime}(\alpha)=[F]$ and $\psi_{0}=\psi_{0}^{\prime} \mid \mathscr{S}^{+}\left(M, \nu_{\varphi}\right)$.

Lemma 4. $\psi_{0}^{\prime}$ and $\psi_{0}$ are well defined. $\psi_{0}$ is additive.

Proof. Since $\psi_{0}^{\prime}, \psi_{0}$ are clearly well defined, it suffices to show that $\psi_{0}$ is additive. Let $\alpha_{i} \in S\left({ }^{+} M, v_{\varphi}\right)$. It follows from the definitions that $\psi_{0}\left(\alpha_{1}+\alpha_{2}\right)=\psi_{0}^{\prime}\left(\alpha_{1} \# \alpha_{2}\right)$ $=\psi_{0}^{\prime}\left(\alpha_{1}\right)+\psi_{0}^{\prime}\left(\alpha_{2}\right)=\psi_{0}\left(\alpha_{1}\right)+\psi_{0}\left(\alpha_{2}\right)$. Therefore $\psi_{0}$ is additive and the lemma is proved.

We conclude this section with the definition of two more maps. First, note that we can identify $H_{n+k} T\left(v_{\varphi}\right)$ with the integers $Z$ in such a way that 1 corresponds to $[M] \in H_{n} M^{n}$ via the Thom isomorphism. Let

$$
\operatorname{deg}: \pi_{n+k} T\left(\nu_{\varphi}\right) \rightarrow H_{n+k} T\left(\nu_{\varphi}\right)
$$

be the Hurewicz homomorphism and define an additive map deg: $\mathscr{S}^{+}\left(M, v_{\varphi}\right) \rightarrow Z$ by $\operatorname{deg}(F, V, f)=\operatorname{deg} f$. Then we have a commutative diagram

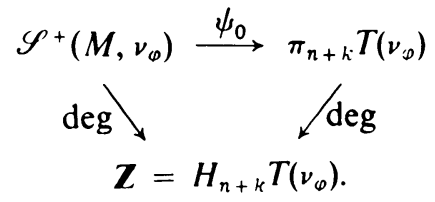


3. The group $\theta\left(M, \nu_{\varphi}\right)$. Let $\alpha, \beta \in \mathscr{S}^{+}\left(M, \nu_{\varphi}\right)$ and set $\varepsilon=\left(T_{\varphi}, M\right.$, identity). Define $\alpha \sim_{e} \beta$ if $\alpha+r \varepsilon=\beta+s \varepsilon$ for some nonnegative integers $r$ and $s$. Obviously, $\sim_{e}$ is an equivalence relation and we can define $\theta\left(M, v_{\varphi}\right)=\mathscr{S}^{+}\left(M, v_{\varphi}\right) / \sim_{e}$. We shall write $[\alpha]$ for the equivalence class in $\theta\left(M, v_{\varphi}\right)$ determined by $\alpha \in \mathscr{S}^{+}\left(M, v_{\varphi}\right)$.

Define an operation + in $\theta\left(M, \nu_{\varphi}\right)$ by $[\alpha]+[\beta]=[\alpha+\beta]$. It is easy to check that + is a well defined associative and commutative operation. $[\varepsilon]$ acts as a zero element. The projection $\mathscr{S}^{+}\left(M, v_{\varphi}\right) \rightarrow \theta\left(M, v_{\varphi}\right)$ is additive.

At this point the only thing which keeps $\theta\left(M, v_{\varphi}\right)$ from being a group is that we do not know whether every element has an inverse. We shall return to this question shortly.

Define $P_{n}$ as usual by

$$
\begin{aligned}
P_{n} & =0 & & \text { if } n \text { is odd } \\
& =Z_{2} & & \text { if } n \equiv 2(\bmod 4) \\
& =Z & & \text { if } n \equiv 0(\bmod 4)
\end{aligned}
$$

We shall think of $P_{n}$ as the set of framed cobordism classes $\left[U^{n}, \mathscr{F}\right]$ of pairs $\left(U^{n}, \mathscr{F}\right)$, where $(U, \partial U) \subseteq\left(D^{n+l}, S^{n+l-1}\right), l \geqq 3, \mathscr{F}$ is a framing of the normal disk bundle of $U$, and $\partial U$ is a homotopy $(n-1)$-sphere (see [10]).

Suppose that $\alpha_{i}=\left(F_{i}, V_{i}, f_{i}\right) \in \mathscr{S}^{+}\left(M, v_{\varphi}\right)$ and that $\psi_{0}\left(\alpha_{1}\right)=\psi_{0}\left(\alpha_{2}\right)$. Then there is a map $H: S^{n+k} \times[1,2] \rightarrow T\left(\nu_{\varphi}\right)$ which is transverse regular on $M$ such that $H \mid S^{n+k} \times i=F_{i} \times i$. Set $W^{n+1}=H^{-1}(M)$ and $h=H \mid W$. Let us try to make $W$ into an $h$-cobordism just as in Lemma 2 . The only problem occurs when we try to do surgery in the middle dimension $[(n+1) / 2]$. This difficulty was circumvented in Lemma 2 by our conditions on $M$ and $n$. But it follows from by now standard techniques that the obstruction to doing this surgery is a well defined element $\gamma\left(\alpha_{1}, \alpha_{2}\right) \in P_{n+1}$. In fact, we may assume that $W$ is diffeomorphic to $V_{2} \times[1,2)$ $\pm U^{n+1}$, where \pm denotes the boundary connected sum along $V_{2} \times 1$ and $(U, \partial U)$ $\subseteq\left(S^{n+k} \times[1,2], S^{n+k} \times 1\right)$ is a $([(n+1) / 2]-1)$-connected $\pi$-manifold with $\partial U$ a homotopy sphere and a framing $\mathscr{F}$ of its normal disk bundle which is induced by $H$ (see $\left[8\right.$, p. 20]). Then $\gamma\left(\alpha_{1}, \alpha_{2}\right)=[U, \mathscr{F}]=\gamma(U, \mathscr{F})$ (see $[10, \S 4.5]$ for a definition of $\gamma(U, \mathscr{F}))$.

Let $\left(U_{1}, \mathscr{F}_{1}\right)$ be a disjoint copy of $(U, \mathscr{F})$ so that $\left(U_{1}, \partial U_{1}\right) \subseteq\left(S^{n+k} \times[1,2]\right.$, $\left.S^{n+k} \times 1\right)$. Define $W \pm-U_{1}$ in a natural manner, where we take the boundary connected sum along $V_{1} \subseteq \partial W$. It is easy to obtain a map $H_{1}: S^{n+k} \times[1,2] \rightarrow T\left(v_{\varphi}\right)$ which is transverse regular on $M$ such that $H_{1}^{-1}(M)=W \pm-U_{1}$ and $H_{1} \mid S^{n+k} \times 2$ $=F_{2} \times 2$. $\left(H_{1}\right.$ is gotten by a construction similar to the one found in the definition of the connected sum of an element of $\mathscr{T}^{+}\left(M, \nu_{\varphi}\right)$ with a framed homotopy sphere.) If we let $F_{3} \times 1=H_{3} \mid S^{n+k} \times 1, V_{3}=F_{3}^{-1}(M)$, and $f_{3}=F_{3} \mid V_{3}$, then we can also assume that $\left(F_{3}, V_{3}, f_{3}\right)=\alpha_{1} \#-\left(\partial U_{1}, \mathscr{F}_{1} \mid \partial U_{1}\right)$. But there is no longer any obstruction to making $W \pm-U_{1}$ into an $h$-cobordism since $\gamma\left((U, \mathscr{F}) \pm-\left(U_{1}, \mathscr{F}_{1}\right)\right)=\gamma(U, \mathscr{F})$ $-\gamma\left(U_{1}, \mathscr{F}_{1}\right)=0$ (see $\left.[10, \S 4.5]\right)$. 
We summarize this discussion in a lemma. Let $\partial_{1}: P_{n+1} \rightarrow \theta_{f}^{n+k, n}$ be given by $\partial_{1}([U, \mathscr{F}])=[\partial U, \mathscr{F} \mid \partial U] . \partial_{1}$ is a homomorphism. This was essentially proved in $[10]$.

Lemma 5. Let $\alpha_{i} \in \mathscr{S}^{+}\left(M, \nu_{\varphi}\right)$ and suppose that $\psi_{0}\left(\alpha_{1}\right)=\psi_{0}\left(\alpha_{2}\right)$. Then

$$
\alpha_{1} \# \partial_{1}\left(\gamma\left(\alpha_{1}, \alpha_{2}\right)\right)=\alpha_{2} \text {. }
$$

Next, let

$$
\pi_{n+k}^{0} T\left(\nu_{\varphi}\right)=\text { kernel of deg: } \pi_{n+k} T\left(\nu_{\varphi}\right) \rightarrow H_{n+k} T\left(\nu_{\varphi}\right),
$$

and define $\psi: \theta\left(M, \nu_{\varphi}\right) \rightarrow \pi_{n+k}^{0} T\left(\nu_{\varphi}\right)$ by $\psi([\alpha])=\psi_{0}(\alpha)-(\operatorname{deg} \alpha) \psi_{0}(\varepsilon)$.

LEMMA 6. $\psi$ is a well defined additive map.

Proof. Suppose that $[\alpha]=[\beta]$. Then $\alpha+r \varepsilon=\beta+s \varepsilon$ for some nonnegative integers $r$ and $s$. Hence $\psi_{0}(\alpha)+r \psi_{0}(\varepsilon)=\psi_{0}(\alpha+r \varepsilon)=\psi_{0}(\beta+s \varepsilon)=\psi_{0}(\beta)+s \psi_{0}(\varepsilon)$ and $\operatorname{deg} \alpha+r$ $=\operatorname{deg}(\alpha+r \varepsilon)=\operatorname{deg}(\beta+s \varepsilon)=\operatorname{deg} \beta+s$. It follows that

$$
\begin{aligned}
\psi_{0}(\alpha)-(\operatorname{deg} \alpha) \psi_{0}(\varepsilon) & =\psi_{0}(\alpha)-s \psi_{0}(\varepsilon)+(s-\operatorname{deg} \alpha) \psi_{0}(\varepsilon) \\
& =\psi_{0}(\beta)-r \psi_{0}(\varepsilon)+(s-\operatorname{deg} \alpha) \psi_{0}(\varepsilon) \\
& =\psi_{0}(\beta)-(\operatorname{deg} \beta) \psi_{0}(\varepsilon)+(\operatorname{deg} \beta-\operatorname{deg} \alpha+s-r) \psi_{0}(\varepsilon) \\
& =\psi_{0}(\beta)-(\operatorname{deg} \beta) \psi_{0}(\varepsilon)
\end{aligned}
$$

and so $\psi$ is well defined. Clearly $\psi(0)=0$.

Let $[\alpha],[\beta] \in \theta\left(M, v_{\varphi}\right)$. Then $\psi([\alpha]+[\beta])=\psi([\alpha+\beta])=\psi_{0}(\alpha+\beta)-(\operatorname{deg}(\alpha+\beta)) \psi_{0}(\varepsilon)$ $=\psi_{0}(\alpha)+\psi_{0}(\beta)-(\operatorname{deg} \alpha) \psi_{0}(\varepsilon)-(\operatorname{deg} \beta) \psi_{0}(\varepsilon)=\psi([\alpha])+\psi([\beta])$. Thus $\psi$ is additive and Lemma 6 is proved.

Note. If $\theta\left(M, v_{\varphi}\right)$ is a group, then $\psi$ is in fact a homomorphism.

Define $\partial_{0}: P_{n+1} \rightarrow \mathscr{S}^{+}\left(M, \nu_{\varphi}\right)$ by $\partial_{0}(\gamma)=\varepsilon \# \partial_{1}(\gamma)$, and let $\partial: P_{n+1} \rightarrow \theta\left(M, \nu_{\varphi}\right)$ be the composition of $\partial_{0}$ followed by the projection of $\mathscr{S}^{+}\left(M, v_{\varphi}\right)$ onto $\theta\left(M, v_{\varphi}\right)$.

LEMMA 7. $\partial_{0}$ and $\partial$ are well defined maps. $\partial$ is a homomorphism.

Proof. $\partial_{0}$ and $\partial$ are well defined because \# is well defined. Let $\gamma_{i} \in P_{n+1}$. Then Lemma 3 implies that

$$
\begin{aligned}
\partial\left(\gamma_{1}+\gamma_{2}\right) & =\left[\partial_{0}\left(\gamma_{1}+\gamma_{2}\right)\right]=\left[\varepsilon \# \partial_{1}\left(\gamma_{1}+\gamma_{2}\right)\right]=\left[\varepsilon \# \partial_{1}\left(\gamma_{1}+\gamma_{2}\right)+\varepsilon\right] \\
& =\left[\varepsilon \# \partial_{1}\left(\gamma_{1}\right)+\varepsilon \# \partial_{1}\left(\gamma_{2}\right)\right]=\left[\varepsilon \# \partial_{1}\left(\gamma_{1}\right)\right]+\left[\varepsilon \# \partial_{1}\left(\gamma_{2}\right)\right] \\
& =\partial\left(\gamma_{1}\right)+\partial\left(\gamma_{2}\right),
\end{aligned}
$$

i.e., $\partial$ is a homomorphism.

Finally, let $\mu: \pi_{n+k}^{0} T\left(\nu_{\varphi}\right) \rightarrow P_{n}$ be the well-known mapping which assigns to every $x \in \pi_{n+k}^{0} T\left(\nu_{\varphi}\right)$ the surgery obstruction to finding a representative $F: S^{n+k}$ $\rightarrow T\left(\nu_{\varphi}\right)$ for $x+\psi_{0}(\varepsilon) \in \pi_{n+k} T\left(\nu_{\varphi}\right)$ such that $F$ is transverse regular on $M$ and $F \mid F^{-1}(M): F^{-1}(M) \rightarrow M$ is a homotopy equivalence. If $n \neq 2(\bmod 4)$, then $\mu=0$. If $n \equiv 2(\bmod 4)$, then our knowledge of $\mu$ is in general limited (see [3]); however, 
with our restrictions on the homology of $M, \mu$ is a well defined homomorphism. Consider the diagram

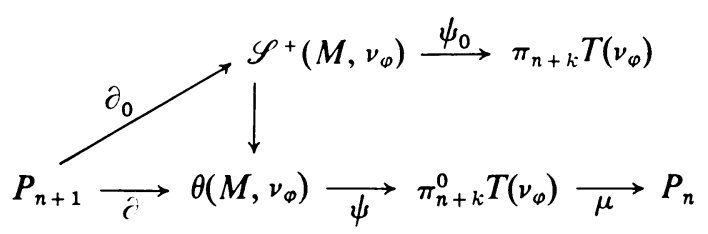

THEOREM 1. $\theta\left(M, v_{\varphi}\right)$ is an abelian group and the bottom row is exact.

Proof. It is easy to see that $\psi_{0} \partial_{0}=0$. From this it follows immediately that $\psi \partial=0$, i.e. (image $\partial) \subseteq(\operatorname{kernel} \psi)$. Let $[\alpha] \in(\operatorname{kernel} \psi)$ and let $\operatorname{deg} \alpha=d$. Then $\psi_{0}(\alpha)=d \psi_{0}(\varepsilon)=\psi_{0}(d \varepsilon)$. By Lemma 5 ,

$$
(d-1) \varepsilon+\partial_{0}(\gamma(d \varepsilon, \alpha))=(d-1) \varepsilon+\left(\varepsilon \# \partial_{1}(\gamma(d \varepsilon, \alpha))\right)=d \varepsilon \# \partial_{1}(\gamma(d \varepsilon, \alpha))=\alpha .
$$

Therefore, $\partial(\gamma(d \varepsilon, \alpha))=[\alpha]$, and we have shown that (kernel $\psi) \subseteq($ image $\partial)$. This proves that $($ image $\partial)=($ kernel $\psi)$.

Next, let $x \in \pi_{n+k}^{0} T\left(\nu_{\varphi}\right)$. Suppose that $\mu(x)=0$. Then $x+\psi_{0}(\varepsilon) \in \pi_{n+k} T\left(\nu_{\varphi}\right)$ belongs to the image of $\psi_{0}$, i.e., there is an $\alpha \in \mathscr{S}^{+}\left(M, \nu_{\varphi}\right)$ with $\psi_{0}(\alpha)=x+\psi_{0}(\varepsilon)$. Hence, $\psi([\alpha])=\psi_{0}(\alpha)-(\operatorname{deg} \alpha) \psi_{0}(\varepsilon)=x$, so that $($ kernel $\mu) \subseteq($ image $\psi)$. Conversely, let $[\alpha] \in \theta\left(M, \nu_{\varphi}\right)$ and set $y=\psi_{0}(\alpha)-(\operatorname{deg} \alpha-1) \psi_{0}(\varepsilon) \in \pi_{n+k} T\left(\nu_{\varphi}\right)$. Then $\operatorname{deg} y=1$. Assume $n \equiv 2(\bmod 4)$. By definition, $\mu(\psi([\alpha]))$ is the obstruction to finding a representative $F: S^{n+k} \rightarrow T\left(\nu_{\varphi}\right)$ for $y$ such that $F$ is transverse regular on $M$ and $F \mid F^{-1}(M): F^{-1}(M) \rightarrow M$ is a homotopy equivalence. But using our hypothesis on the homology of $M$, we see that for this particular $y$ we can start with a representative $F$ such that $H_{t}\left(F^{-1}(M)\right)=0$ and $H_{t-1}\left(F^{-1}(M)\right)$ is torsion-free, where $t=[n / 2]$. Therefore, we shall never have to do surgery in the middle dimension; and so $\mu(\psi([\alpha]))=0$. Since $\mu=0$ when $n \not \equiv 2(\bmod 4)$, we have shown that (image $\psi)$ $=(\operatorname{kernel} \mu)$.

It remains to prove that $\theta\left(M, v_{\varphi}\right)$ is an abelian group. As was observed earlier, it suffices to show that every $a \in \theta\left(M, \nu_{\varphi}\right)$ has an inverse. Choose a $b \in \psi^{-1}(-\psi(a))$. Such a $b$ exists because (image $\psi)=\left(\right.$ kernel $\mu$ ) is a subgroup of $\pi_{n+k}^{0} T\left(\nu_{\varphi}\right)$. Then $\psi(a+b)=\psi(a)+\psi(b)=\psi(a)-\psi(a)=0$. By exactness we can now find a $\gamma \in P_{n+1}$ such that $\partial(\gamma)=a+b$. Hence $0=\partial(0)=\partial(\gamma-\gamma)=\partial(\gamma)+\partial(-\gamma)=a+(b+\partial(-\gamma))$. This finishes the proof of Theorem 1 .

Let us show that Theorem 1 is a generalization of a well known exact sequence. Suppose that $M^{n}=S^{n}$ and $\varphi: S^{n} \rightarrow S^{n+k}$ is the standard inclusion. Define $\lambda_{0}: \mathscr{S}^{+}\left(S^{n}, \nu_{\varphi}\right) \rightarrow \theta_{f}^{n+k, n}$ by $\lambda_{0}((F, V, f))=\left[V, \mathscr{F}_{F}\right]$, where $\mathscr{F}_{F}$ is the framing of $V$ induced from the framing of $S^{n}$ in $S^{n+k}$. (Note that $V$ is indeed a homotopy sphere.) Clearly $\lambda_{0}$ is well defined. Also, $\lambda_{0}(\alpha+r \varepsilon)=\lambda_{0}(\alpha)$ for each $\alpha \in \mathscr{S}^{+}\left(S^{n}, \nu_{\varphi}\right)$ because if $\mathscr{F}_{0}$ is the standard framing of $\nu_{\varphi}$, then $\left(\Sigma^{n}, \mathscr{F}\right) \#\left(S^{n}, \mathscr{F}_{0}\right)=\left(\Sigma^{n}, \mathscr{F}\right)$ for every framed homotopy sphere $\left(\Sigma^{n}, \mathscr{F}\right)$ in $S^{n+k}$. (Here \# denotes the operation 
of framed connected sum which induces the addition in $\theta_{f}^{n+k, n}$.) Therefore, $\lambda_{0}$ induces a well-defined map $\lambda: \theta\left(S^{n}, \nu_{\varphi}\right) \rightarrow \theta_{f}^{n+k, n}$ given by $\lambda([\alpha])=\lambda_{0}(\alpha)$.

If $[\Sigma, \mathscr{F}] \in \theta_{f}^{n+k, n}$, let $f: \Sigma \rightarrow S^{n}$ be a homotopy equivalence with $\operatorname{deg} f=1$. Let $g: \nu_{\Sigma} \rightarrow \nu_{\varphi}$ be given by $g(\mathscr{F}(y, u))=(h(y), u) \in S^{n} \times D^{k} \subseteq S^{n+k}$ for $(y, u) \in \Sigma \times D^{k}$. Then $g$ induces a map $F: S^{n+k} \rightarrow T\left(\nu_{\varphi}\right)$, and $\lambda_{0}((F, \Sigma, f))=[\Sigma, \mathscr{F}]$. Thus $\lambda_{0}$, and hence $\lambda$ is onto.

Next, let $\alpha, \beta \in \mathscr{S}^{+}\left(S^{n}, \nu_{\varphi}\right)$ and suppose that $\lambda_{0}(\alpha)=\lambda_{0}(\beta)$. Without loss of generality assume that $\operatorname{deg} \beta-\operatorname{deg} \alpha=r \geqq 0$. Then it is not hard to show that $\alpha+r \varepsilon=\beta$. (Observe that if $\alpha_{i} \in \mathscr{S}^{+}\left(S^{n}, \nu_{\varphi}\right)$, then $\alpha_{1}+\alpha_{2}=\alpha_{1} \# \alpha_{2}$.) It follows that $\lambda$ is one-to-one. But $\lambda_{0}$, and hence $\lambda$, is additive since the addition in $\mathscr{S}^{+}\left(S^{n}, v_{\varphi}\right)$ and $\theta_{f}^{n+k, n}$ both come from a connected sum operation, and so we have proved

LEMMA 8. $\lambda$ is an isomorphism.

Now $\nu_{\varphi}$ is trivial, and so $T\left(\nu_{\varphi}\right)=S^{n+k} \vee S^{k}$ by [11]. $\pi_{n+k} T\left(\nu_{\varphi}\right)=\pi_{n+k} S^{n+k}$ $\oplus \pi_{n+k} S^{k}$, where deg maps the first factor isomorphically onto $H_{n+k} T\left(\nu_{\varphi}\right)=Z$. Therefore, we can identify $\pi_{n+k}^{0} T\left(\nu_{\varphi}\right)$ in a natural way with $\pi_{n+k} S^{k}$. (In fact, one can make this identification in the case of any imbedding $\varphi: S^{n} \rightarrow S^{n+k}$ with $2 k \geqq n+3$ because $v_{\varphi}$ will then be trivial by [6].) It follows from Lemma 8 that the sequence

$$
P_{n+1} \underset{\partial}{\longrightarrow} \theta\left(S^{n}, \nu_{\varphi}\right) \underset{\psi}{\longrightarrow} \pi_{n+k}^{0} T\left(\nu_{\varphi}\right) \underset{\mu}{\longrightarrow} P_{n}
$$

can be identified with the Milnor-Kervaire sequence

$$
P_{n+1} \underset{\partial_{1}}{\longrightarrow} \theta_{f}^{n+k, n} \underset{\psi_{1}}{\longrightarrow} \pi_{n+k} S^{k} \underset{\mu_{1}}{\longrightarrow} P_{n},
$$

where $\psi_{1}$ is defined via the Pontrjagin-Thom construction and $\mu_{1}$, like $\mu$, is the usual surgery obstruction.

These observations lead us to another definition of $\theta\left(M, v_{\varphi}\right)$. Briefly, it is possible to define $\theta\left(M, v_{\varphi}\right)$ to be the $h$-cobordism classes of $(F, V, f)$ for which $f$ is a homotopy equivalence. The sum of $\left[\left(F_{1}, V_{1}, f_{1}\right)\right]$ and $\left[\left(F_{2}, V_{2}, f_{2}\right)\right]$ is defined to be the class of that triple $\left(F_{3}, V_{3}, f_{3}\right)$ which is obtained from $\left(F_{1}, V_{1}, f_{1}\right) \#\left(F_{2}, V_{2}, f_{2}\right)$ \# $\left(T_{\varphi},-M\right.$, identity) by surgery for which $f_{3}$ is a homotopy equivalence. In order that this addition is well defined and that we get a group we have to be able to do the necessary surgery. This is why we need some conditions on $n$ and the homology of $M$. Our condition, that either $n \equiv 0(\bmod 4)$ or $H_{t} M=0$ and $H_{t-1} M$ is torsionfree, can probably be weakened. The reason that we did not give this straightforward definition of $\theta\left(M, v_{\varphi}\right)$ at the beginning and proceeded in a roundabout fashion to define $\mathscr{S}^{+}\left(M, v_{\varphi}\right)$ first is that $\mathscr{S}^{+}\left(M, v_{\varphi}\right)$ and $\psi_{0}$ are interesting in their own right (see the next section).

Next, observe that the inclusion $i: S^{n+k} \subseteq S^{n+k+1}$ induces natural maps $\mathscr{T}_{0}^{+}\left(M, v_{\varphi}\right) \rightarrow \mathscr{T}_{0}^{+}\left(M, v_{i \varphi}\right), \mathscr{S}_{0}^{+}\left(M, v_{\varphi}\right) \rightarrow \mathscr{S}_{0}^{+}\left(M, \nu_{i \varphi}\right), \mathscr{T}^{+}\left(M, v_{\varphi}\right) \rightarrow \mathscr{T}^{+}\left(M, v_{i \varphi}\right)$, $\mathscr{S}^{+}\left(M, v_{\varphi}\right) \rightarrow \mathscr{S}^{+}\left(M, v_{i \varphi}\right)$, and $\theta\left(M, \nu_{\varphi}\right) \rightarrow \theta\left(M, v_{i \varphi}\right)$. These "suspension" maps 
are clearly additive and will all be denoted by $s$. Let $\theta^{k}$ be the trivial $k$-disk bundle over $M$. Then $\nu_{i \varphi}=\nu_{\varphi} \oplus \theta^{1}$, and the following diagrams commute:
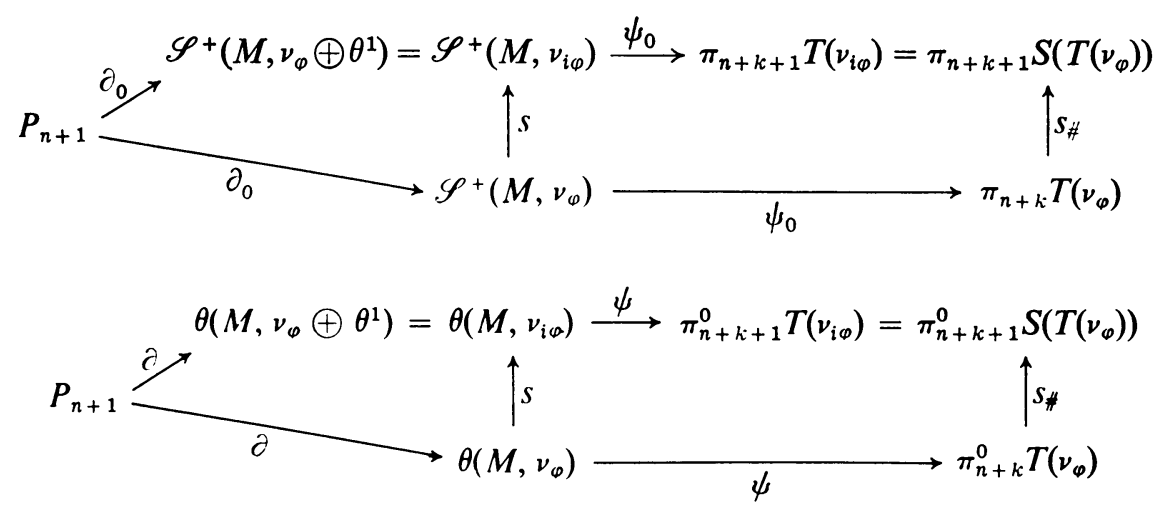

$\left(S\left(T\left(v_{\varphi}\right)\right)\right.$ is the reduced suspension of $T\left(\nu_{\varphi}\right)$ and $s_{\#}$ is the usual suspension map on homotopy.)

Finally, define

$$
\theta(M)=\lim _{t} \theta\left(M, \nu_{\varphi} \oplus \theta^{t}\right)
$$

It follows from the above remarks and Theorem 2 that $\theta(M)$ is a well-defined abelian group. In fact, $\theta(M)$ is isomorphic to $\theta\left(M, \nu_{\varphi} \oplus \theta^{t}\right)$ whenever $k+t \geqq n+3$. $\theta(M)$ is the group of manifolds which are "framed" homotopy equivalent to $M$.

4. Conclusion. We would like to conclude with some unanswered questions which arise naturally in the context of this paper:

1. $\theta\left(M, v_{\varphi}\right)$ corresponds to $\theta_{f}^{n+k, n}$. A natural analogue of $\theta^{n+k, n}$, the $h$-cobordism classes of (unframed) homotopy $n$-spheres in $S^{n+k}$, would seem to be the set, $\mathscr{S}_{k}(M)$, of $h$-cobordism classes of homotopy smoothings of $M$ which are imbedded in $S^{n+k}$. The set $\mathscr{S}_{k}(M)$, for large $k$, was considered in [12] and fit into an exact sequence. There is a commutative diagram

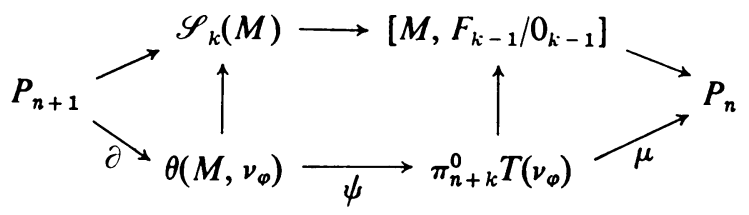

The top row was defined in [12] for large $k$ and shown to be exact. It reduces to the Milnor-Kervaire sequence when $M^{n}=S^{n}$. Can one generalize other sequences in [5] and [10]?

2. When is an element $x \in \pi_{n+k} T\left(\nu_{\varphi}\right)$ in the image of $\psi_{0}$ ? This question is partially answered in [2] and involves the study of Browder-Novikov theory for maps of degree $d>1$. Note how much easier it is to determine the image of $\psi$. 
3. Let $(F, V, f) \in \mathscr{S}^{+}\left(M, v_{\varphi}\right)$. Is $V$ homotopy equivalent to $M$ ? (The homotopy equivalence may have no relation to $f$.) This question in conjunction with question 2 has bearing on the problem of whether manifolds imbed in the metastable range. Since $f_{*}\left(f^{*}(a) \cap[V]\right)=(\operatorname{deg} f)(a \cap[M])$ for $a \in H^{i} M$, it follows that $f_{*}: H_{i} V \rightarrow H_{i} M$ is an isomorphism for $0 \leqq i<n$ whenever $H_{i} M$ is finite and the order of $H_{i} M$ is relatively prime to $\operatorname{deg} f$ for $0<i<n$. This suggests a somewhat weaker question: Is $V$ homotopy equivalent to $M$ if $f_{*}: H_{i} V \rightarrow H_{i} M$ is an isomorphism for $0 \leqq i<n$ ?

4. When does a manifold $M^{n}$ admit a map $f: M \rightarrow M$ of degree $d>0$ ? Are there some more or less simple conditions on the homology or homotopy groups which will guarantee the existence of $f$ ? This problem fits into our context because it is related to the previous questions about $\mathscr{S}^{+}\left(M, v_{\varphi}\right)$ and $\pi_{n+k} T\left(\nu_{\varphi}\right)$.

5. Would it be useful to study manifolds $M^{n}$ which have the property that $d \varepsilon=\left(F_{d}, \varphi\left(M^{n}\right), f_{d}\right)$ ? For example, $M^{n}=S^{n}$ has this property. Do products of spheres $S^{l} \times S^{n-l}$ behave similarly?

\section{REFERENCES}

1. M. K. Agoston, S-tangential maps and imbeddings in the conditionally stable range, Topology 9 (1970), 13-23.

2. - Browder-Novikov theory for maps of degree d > 1. I, Topology (3) 9 (1970), 251-266.

3. W. Browder, The Kervaire invariant of framed manifolds and its generalization, Ann. of Math. (2) 90 (1969), 157-186.

4. A. Haefliger, Knotted (4k-1)-spheres in 6k-space, Ann. of Math. (2) 75 (1962), 452-466. MR 26 \#3070.

5. - Differentiable embeddings of $S^{n}$ in $S^{n+q}$ for $q>2$, Ann. of Math. (2) 83 (1966), 402-436. MR 34 \#2024.

6. M. A. Kervaire, An interpretation of $G$. Whitehead's generalization of $H$. Hopf's invariant, Ann. of Math. (2) 69 (1959), 345-365. MR 21 \#1595.

7. M. A. Kervaire and J. Milnor, Groups of homotopy spheres. I, Ann. of Math. (2) 77 (1963), 504-537. MR $26 \# 5584$.

8. R. Lashof, Some theorems of Browder and Novikov on homotopy equivalent manifolds with an application, Univ. of Chicago Notes, prepared by R. DeSapio.

9. J. Levine, On differentiable imbeddings of simply-connected manifolds, Bull. Amer. Math. Soc. 69 (1963), 806-809. MR 27 \#5270.

10. — A classification of differentiable knots, Ann. of Math. (2) 82 (1965), 15-50. MR 31 \#5211.

11. S. P. Novikov, Homotopically equivalent smooth manifolds. I, Izv. Akad. Nauk SSSR Ser. Mat. 28 (1964), 365-474; English transl., Amer. Math. Soc. Transl. (2) 48 (1965), 271-396. MR 28 \#5445.

12. D. Sullivan, Smoothing homotopy equivalences, Notes, University of Warwick, Coventry.

WESLEYAN UNIVERSITY, Middletown, ConNecticut 06457 